\title{
La aspirina en bajas dosis no previene la preeclampsia en las mujeres con embarazo de alto riesgo
}

Low-dose aspirin to prevent preeclampsia in women at high risk.

Caritis S, Sibai B, Hauth J, Lindheimer MD et al. N Engl J Med 1998;338:701-5.

\section{Objetivo}

Determinar si la terapéutica con aspirina reduce la incidencia de preeclampsia en mujeres con elevado riesgo para esta enfermedad.

\section{Diseño}

Estudio prospectivo randomizado doble ciego controlado con placebo.

\section{Lugar}

Departamentos de Obstetricia y Ginecología de 10 Universidades de EE.UU.

\section{Pacientes}

Participaron 2539 embarazadas: 471 mujeres con diabetes mellitus pregestacional tratadas con insulina, 774 mujeres con hipertensión crónica, 688 mujeres con embarazo múltiple y 606 mujeres que habían tenido preeclampsia durante un embarazo previo.

\section{Intervención}

Las mujeres fueron incluidas entre las $13^{a}$ y $26^{a}$ semanas de gestación y recibieron $60 \mathrm{mg} /$ día de aspirina o placebo.

\section{Medición de resultados principales}

Se registraron los casos de preeclampsia en cada uno de los grupos. Los resultados del seguimiento fueron obtenidos en 2503 de las 2539 mujeres que entraron en el estudio.

\section{Resultados principales}

La incidencia de preeclampsia fue similar entre las 1254 mujeres del grupo tratado con aspirina y entre las 1249 mujeres del grupo placebo (grupo aspirina: 18\%; grupo placebo, $20 \%$; $P=0,23$ ).Las incidencias de preeclampsia fueron similares para cada una de las cuatro categorías de alto riesgo; para las mujeres con diabetes mellitus pregestacional la incidencia fue del $18 \%$ en el grupo aspirina y $22 \%$ en el grupo placebo $(\mathrm{P}=0,38)$; para las mujeres con hipertensión crónica, $26 \%$ y $25 \%(\mathrm{P}=0,66)$; para aquellas con embarazos múltiples, $12 \%$ y $16 \%(P=0,10)$; y para aquellas con preeclampsia durante un embarazo previo, $17 \%$ y $19 \%(P=0,47)$.

Además, las incidencias de muerte perinatal, nacimiento prematuro, y recién nacidos pequeños para la edad gestacional fueron similares entre los grupos aspirina y placebo.

\section{Conclusiones}

La aspirina en bajas dosis no reduce la incidencia de preeclampsia ni mejora el pronóstico perinatal en mujeres con alto riesgo de desarroLlar preeclampsia.

\section{COMENTARIO}

Este estudio sirve para continuar la controversia respecto a la posibilidad de la prevención de preeclampsia con aspirina a bajas dosis. La mayoría de los pequeños estudios que evaluaron la aspirina a bajas dosis realizados en mujeres con elevado riesgo para desarrollar preeclampsia, mostraban una reducción sustancial en el riesgo de hipertensión arterial con proteinuria y una reducción en la incidencia de partos con recién nacidos prematuros, de bajo peso para la edad gestacional y de muerte perinatal.(1-3) Estos trabajos llevaron a una amplia utilización de la aspirina para prevenir la preeclampsia. Con el tiempo, algunos grandes ensayos no revelaron beneficios con el uso de aspirina.(4-6) Por otra parte, en la mayoría de los estudios en realidad se incluyeron miujeres con bajo riesgo para desarrollar preeclampsia, lo cual se evidencia por la baja incidencia $(2,5 \%$ a $7,6 \%)$ de preeclampsia en las mujeres que recibieron placebo. Resulta evidente la disparidad entre los pequeños estudios (menos de 200 mujeres) y los gran- des ensayos (más de 200 mujeres). En los primeros, la terapéutica antiplaquetaria se asoció con una reducción del $82 \%$ en el riesgo de desarrollar preeclampsia, mientras que en los últimos dicha reducción fue solo del 9\%. Una explicación probable podría ser el sesgo de publicación, ya que los pequeños estudios con resultados positivos son probablemente los que más se envían a las revistas y los que tienen más posibilidad de publicarse a diferencia de los pequeños ensayos con resultados ambiguos o negativos (7).

Cuando se combinan los pequeños y los grandes estudios, y sin tener en cuenta el sesgo de publicación, la aspirina reduciría la incidencia de preeclampsia en un $2.5 \%$, una diferencia estadísticamente significativa pero de cuestionable importancia clínica. Aún en grupos de mujeres de alto riesgo como las incluídas en este último ensayo, deberíamos tratar a 38 mujeres para prevenir un caso de preeclampsia. (NNT)

\section{$\begin{array}{ll}\text { Dr. Gabriel Waisman } & \text { Dran }\end{array}$}

\footnotetext{
Referencias

1. Beaufils M, Uzan S, Donsimoni R, Colau JC. Prevention of pre-eclampsia by early antiplatelet therapy. Lancet 1985;i:840-2.

2. Uzan S, Beaufils M, Breart G, Bazin B et al. Prevention of fetal growth retardation with low-dose aspirin: findings of the EPREDA trial. Lancet 1991;337:1427-31.

3. Hauth JC, Goldenberg RL, Parker CR Jr, et al. Low-dose aspirin therapy to prevent preeclampsia. Am J 0bstet Gynecol 1993;168:1083-91.

4. Sibai BM, Caritis SN, Thom E, et al. Prevention of preeclampsia with low-dose aspirin in healthy, nulliparous pregnant women. N Engl J Med 1993;329:1213-8.

5. CLASP (Collaborative Low-dose Aspirin Study in Pregnancy) Collaborative Group. CLASP: a randomised trial of low-dose aspirin for the prevention and treatment of pre-eclampsia among 9364 pregnant women. Lancet 1994;343:619-29.

6. ECPPA (Estudio Colaborativo para Prevencao da Pre-eclampsia com Aspirina) Collaborative Group. ECPPA: randomised trial of low dose aspirin for the prevention of maternal and fetal complications in high risk pregnant women. Br J Obstet Gynecol 1996;103:39-47.

7. Pipkin B, Crowther C, de Swiet M, et al. Where next for prophylaxis against pre-eclampsia? Br J Obstet Gynaecol 1996;103:603-7.
} 